\title{
Update on the harmonisation of disability data collection in UK surveys (Part 2)
}

Chris White Office for National Statistics

\section{Abstract}

\section{Background}

This article reports on the field testing work undertaken, leading to the proposals for a primary harmonised standard for disability data inputs: designed to derive statistical measures of disability from social surveys using a face-to-face mode of data capture. These proposals were submitted to the National Statistics Harmonisation Group (NSHG) for approval in December 2010. The proposed primary harmonised standard data inputs are designed to meet the data needs arising from the equality legislation introduced in 2010 and improve international comparability: by better meeting the definitions for measures of longstanding illness and disability derived from the European Union's Statistics on Income and Living Conditions (EU-SILC).

\section{Methods}

Field testing was conducted during two discrete periods: between October 2009 and February 2010 for stage 1 testing, and between May and July 2010 for stage 2 testing. The Office for National Statistics (ONS) Opinions Survey acted as the data collection vehicle and the design was a split sample in each case. In stage 1, question suite variants were compared, together with the Family Resources Survey (FRS) Disability Discrimination Act module running on the Opinions Survey in equivalent months. The second stage also applied a split sample to compare the revised proposed harmonised questions with the question on disability planned for the 2011 Census.

Derivations of disability to meet measures required under the national Equality Act legislation and the European Union-Statistics on Income and Living Conditions were applied to field-test estimates to assess coherence with contemporaneous external sources.

\section{Results}

The field test results found good comparability with estimates of disability used in the FRS publications and with the field tested 2011 Census disability question. 
In stage 1 testing, the measure of disability derived from the version 1 suite was closer to the FRS measure of disability used in FRS publications than that derived from version 2's questions. In stage 2, the proximity of the measure derived from the proposed harmonised questions to the FRS measure of disability improved, with a 0.5 percentage point difference.

The stage 2 measure of disability was found to be consistent with the EU-SILC measure of disability in $\mathbf{2 0 0 9}$ for the UK published on the Eurostat website. Furthermore, the harmonised questions produced a similar estimate of disability to the 2011 Census question, also field tested concurrently with the proposed harmonised questions.

\section{Conclusions}

The harmonised questions tested provide appropriate data inputs to measure persistent illness, impairment and disability, consistent with the components of disability used in the disablement process and the International Classification of Functioning Disability and Health. Their implementation across social survey data sources using a face-to-face mode of data capture will enhance the consistency of statistical measurement and their relevance to the data requirements embodied in national Equality legislation and the EU-SILC European regulation. 


\section{Contents}

Abstract.

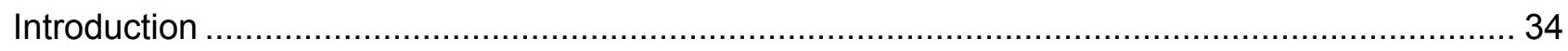

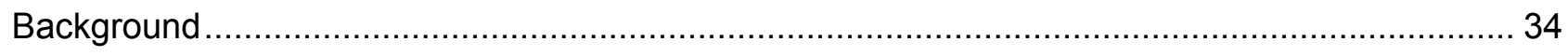

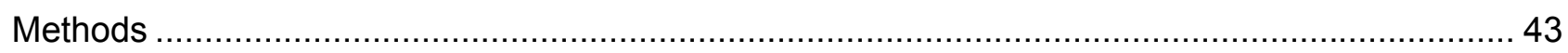

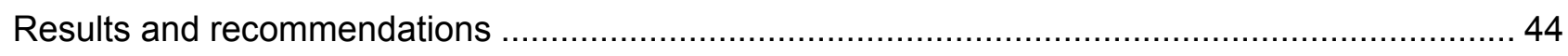

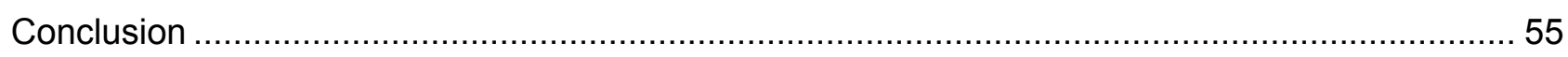

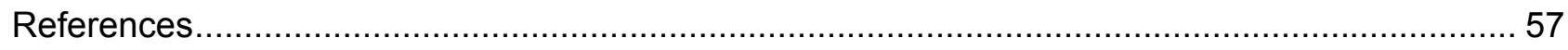

Annex A

Annex B …

Annex C

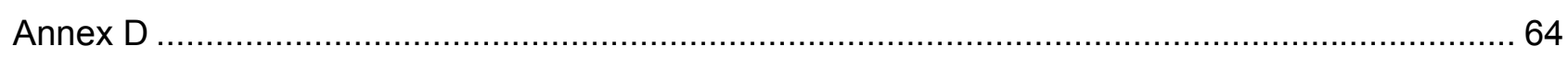

\section{List of Tables}

Table 1 Comparison in percentage prevalence of persistent illness by source, persons aged

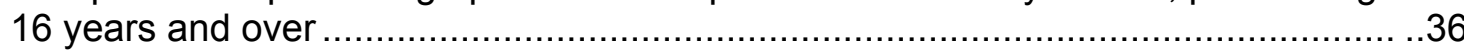

Table 2 Percentage prevalence of impairment by version, persons aged 16 years and over...37

Table 3 Percentage prevalence of activity restriction by source: Persons aged 16 years and

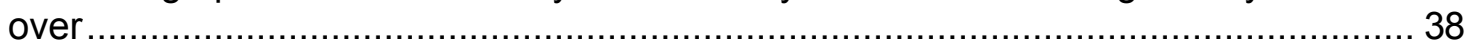

Table 4 Other impairments which collapsed into an existing impairment category …..............40 40

Table 5 Percentage prevalence of impairment in those reporting substantial difficulty in a

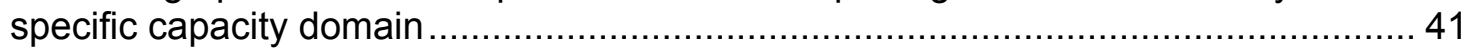

Table 6 Percentage prevalence of a physical or mental health condition: by source ............. 45

Table 7 Percentage prevalence of impairment: by source, persons aged 16 years and over.. 46

Table 8 Percentage prevalence of activity restriction by extent, persons aged 16 years and over.

Table 9 EU-SILC derived disability percentage prevalence, persons aged 16 years and over.. 


\section{Introduction}

This article reports progress made in revising standard UK social survey questions on persistent illness, impairment and activity restriction for collection in social survey data sources using a faceto-face mode of data collection. It updates the material presented in an accompanying article in this issue of HSQ detailing the consultations and cognitive testing components of the project (White 2011). The findings of consultation and cognitive question testing were used to amend the draft questions for field testing, and the evidence gained from field testing enabled the refinement of data inputs to derive statistical measures of disability, which are described below.

\section{Background}

The Review of Equality Data (ONS, 2007) identified a need to improve the co-ordination, comparability, quality, accessibility and presentation of disability statistics through developing and applying a principled approach to data collection and its circulation. It made a specific recommendation that the Office for Disability Issues (ODI) and the Government Equalities Office (GEO) should work with the Office for National Statistics (ONS) to develop harmonised standards for disability measurement across data sources to meet this need.

A project was initiated in early 2008 with the following objectives:

a) conduct a cross-sector consultation on data needs and priorities and replicate consultations at key stages

b) develop a conceptual framework for disability definition taking account of consultation responses, national legislation and European regulations

c) frame a short suite of questions to measure disability in accordance with the definition and sensitive to the issue of data continuity

d) test the performance of these questions in terms of interpretability and comparability with established sources collecting similar information

e) recommend to the National Statistics Harmonisation Group (NSHG) and the Government Statistical Service Statistical Policy and Standards Committee (GSS SPSC) an implementation of harmonised data inputs across social survey sources to harmonise the presentation of statistical measures of disability

This article contains the following sections:

- The results from two stages of field testing

- $\quad$ Final consultation on the implications of these results

- Recommendation of a primary harmonised standard to key fora, including the NSHG and the GSS SPSC.

The article provides a condensed overview of field test results; the finalised field testing reports will be published on the ONS website during the autumn and will be available for downloading. 


\section{Field Testing Stage 1}

\section{Methods}

\section{Data Source and Study population}

ODI commissioned the first field testing project, and used the ONS Opinions Survey (OS) as the data collection vehicle (ONS Opinions Survey, 2011). The OS is multi-purpose, designed to test questions in the field before embedding them into other established data sources; the sampling approach ensures delivery of a representative sample of Great Britain aged 16 years and over, with one household member selected for interview. The data was collected over a five-month period from October 2009 to February 2010.

\section{Question suite versions and design}

Two question suite versions (see Annex A) were tested. The first version asked Disability Discrimination Act (DDA) (National Archive, 1995) related questions, including a question on frequency of activity restriction; but excluded a question on duration. It routed respondents to the impairment list question only if they reported having a persistent health condition or illness in the previous question. The question capturing persistent health conditions and illnesses excluded the terms disability, infirmity and impairment and asked respondents to consider whether they had any physical or mental health conditions or illnesses that have lasted 12 months or more.

The second version asked questions about participation restriction and the perceived causes of restriction first (not reported on here) before asking DDA related questions. Version 2 excluded the capture of frequency of activity restriction but included a question on duration, and the type of impairment question was asked across the whole sample, not routed as in version 1 . The question capturing persistent health conditions and illnesses also excluded the terms disability, infirmity and impairment and asked respondents whether they had any physical or mental health conditions expected to last 12 months or more.

The sample was split in half, with one half administered the version 1 questions and the other half the version 2 questions. The sample size for version 1 was 2,403 and for version 2, was 2,384.

\section{Elements of testing}

The primary purpose of this stage of testing was to judge comparability of estimates of disability derived using each field-tested version with those derived from the FRS's disability module (See Annex B) and estimates of long-standing illness and disability provided by the General Lifestyle Survey's (GLF) questions (See Annex C) designed to capture two variables included in the European Union's Statistics on Income and Living Conditions' (EU-SILC) Minimum European Health Module (MEHM). 
The broad testing elements included:

a) the extent of comparability in component-specific prevalence estimates of illness, impairment and disability between suite versions and external sources, and capability to estimate core current DDA disability and disability derived from the EU-SILC MEHM

b) the performance and adequacy of the impairment list in capturing respondents' impairment

c) the extent of agreement between the proposed harmonised impairment list and the FRS list of capacities

\section{Results and recommendations}

\section{Comparability in component specific prevalence}

The prevalence of persistent illness varied between field test versions, with version 1 producing a 4.2 percentage point higher prevalence than version 2 (Table 1). Table 1 documents the question wording used in each source and tested version, together with the percentage prevalence detected in those aged 16 years and over.

\section{Table $1 \quad$ Comparison in percentage prevalence of persistent illness by source, persons aged 16 years and over}

Source and Question

Family Resources Survey 2008-09

Do you have any long-standing illness, disability or infirmity lasting or expected to last 12 months or more?

Life Opportunities Survey 2009-10

Do you have any long-standing physical or mental impairment, illness or disability lasting or expected to last for a period of 12 months or more? By long-standing I mean anything that has affected you over a period of at least 12 months or that is likely to affect you over a period of at least 12 months.

General Lifestyle Survey 2008

Do you have any long-standing illness, disability or infirmity - by long-standing I mean anything that has troubled you over a period of time or is likely to affect you over a period of time?

Annual Population Survey 2006-08

Do you have any health problems or disabilities that you expect will last for a year or more?

Field Test stage 1 version 1 Opinions 2009-10

Do you have any physical or mental health conditions or illnesses which have lasted for 12 months or more?

Field Test stage 1 version 1 Opinions 2009-10

Do you have any physical or mental health condition(s) or illnesses that are expected to last 12 months or more?

A comparison with external sources showed the field-tested questions produced predominantly lower percentage prevalence, although version 1's question was found to be more proximal than version 2's. The possible risk of suppressing prevalence by excluding the terms disability or impairment from the body of the field tested question and leading to a discontinuity is contentious as the FRS question contains the term disability, but this source produced a lower prevalence compared with the version 1 field test question; however, all other sources investigated, which contained one or other of these terms produced a higher prevalence. 
The field-test questions are more specific in what they capture (that is physical or mental health conditions or illnesses) and they reference only either the past 12 months (version 1) or the future 12 months (version 2), which may contribute to a lower prevalence found, compared with the GLF, LOS and APS questions.

A second component tested was the possible impact on prevalence of specific impairments by routeing the impairment question to only those reporting a physical or mental health condition in question 1, as applied in version 1, compared with total sample coverage applied in version 2 (Table 2).

\section{Table 2 Percentage prevalence of impairment by version, persons aged 16 years and over}

\begin{tabular}{lrr} 
Impairment & Version $\mathbf{1}$ & Version 2 \\
\hline Seeing (due to blindness or partial sight) & 3.5 & 4.2 \\
Hearing (due to deafness or partial hearing) & 4.2 & 6.6 \\
Mobility (difficulty walking short distances, climbing stairs, lifting or carrying objects) & 14.8 & 14.7 \\
Learning or Concentrating or Remembering & 4.1 \\
Mental Health & 3.7 & 6.0 \\
Stamina or Breathing & 7.4 & 3.9 \\
Social learning difficulty (e.g, due to Autistic Spectrum Disorder or Aspergers') & 0.3 & 0.5 \\
Other (please specify) & 4.4 & 2.7 \\
Any impairment & 25.0 & 29.7 \\
\hline
\end{tabular}

Source: ONS Opinions Survey 2009-10

Prevalence of impairment overall was 4.7 percentage points higher using version 2's administration approach, with higher prevalence in the majority of specific categories. The overall version 2 prevalence of 30 per cent concurred well with the overall prevalence found in the Life Opportunities Survey (LOS) interim wave 1 report (ONS, 2010), and suggests a small number of respondents will differentiate between having a health condition and having an impairment.

The prevalence of activity restriction, and thereby the prevalence of disability, using version 1's questions which capture frequency and extent of restriction, was 19.7 per cent, suggesting approximately a fifth of the population were restricted either 'a lot' or 'a little' 'sometimes' or more frequently. In comparison, version 2's question suggested a population prevalence of only 18.9 per cent (Table 3.). 
Table 3 Percentage prevalence of activity restriction by source: Persons aged 16 years and over

Source

Weighted per

cent

Field test version 1 suite.- Opinions 2009-10

To what extent do you have difficulty carrying out day to day activities?

Yes a lot of difficulty or yes a little difficulty?

Yes, a lot of difficulty

Yes, a little difficulty

Field test version 2 suite.- Opinions 2009-10

In relation to your condition(s) or illnesses, to what extent do you have difficulty carrying out day to day activities?

Yes a lot of difficulty or yes a little difficulty?

Yes, a lot

Yes, a little

FRS question - 2008-09

Does this/do these health problems or disabilities mean that you have substantial difficulties with any of these areas of your life?

Mobility; lifting; manual dexterity; continence; communication; memory, concentrate, understand or learn; physical danger; balance; other

LOS question 2009-10 interim results

Does this/do these health problems or disabilities mean that you have substantial difficulties with any of these areas of your life?

Mobility; lifting; manual dexterity; continence; communication; memory, concentrate, understand or learn; physical danger; balance; other

\section{GLF question 2008}

Does this illness or disability (Do any of these illnesses or disabilities) limit your activities in any way? Limited or strongly limited

The routeing approach differed in each version; version 1 respondents were routed from the first question only if answering 'yes'; however, in version 2 respondents were routed if they either reported a health condition or illness in question $2 a$ or an impairment in question $2 b$ (see Annex A).

The lower prevalence detected using version 2's questions could have arisen by misclassifying those with intermittent restrictions because of the absence of a question capturing frequency; however, the lower prevalence of health conditions or illnesses in version 2 is a plausible alternative explanation.

The frequency category with the highest prevalence in version 1's frequency question was 'sometimes' - exceeding the prevalence of the other two categories combined (that is 'always' and 'most of the time'). The scale of this difference suggests the non-capture of those with intermittent restrictions was an unlikely explanation for the lower prevalence detected in version 2 , as it is arguable that the difference in prevalence would have been considerably greater if respondents with intermittent restrictions had been reluctant to affirm activity restriction in version 2's question for this reason. Consequently, there is an absence of clear evidence supporting the need to collect frequency of restriction to avoid the misclassification of those with intermittent restriction effects.

The field-test results refute the perceived risk of suppressing the prevalence estimates of activity restriction, and thereby disability, by routeing to the impairment question. While the prevalence of 
impairment itself is somewhat lower in the routed sample, there is no evidence of a knock-on effect for measures of activity restriction (that is disability), suggesting the non-routed sample is capturing impairments not associated with activity restriction. As it is the impaired population with activity restriction that is the most useful for policy, the additional costs incurred by administrating the impairment question across the whole sample seems unnecessary.

The prevalence of activity restriction is notably lower than LOS. However, compared with the FRS and GLF, on which the questions are benchmarked, the field test questions produce similar estimates.

The perceived risk that the capture of extent of restriction using the plain English response categories 'a lot of difficulty', or 'a little difficulty' would inflate overall prevalence compared with dichotomised response options using 'Yes' and 'No' response categories is not borne out by these field-test results.

\section{Performance and adequacy of the impairment list}

The performance and adequacy of the impairment list was judged on the basis of what proportion of the sample used the 'Other impairment' category, and whether those with a health condition or illness report one or more of the impairment categories.

The proportion of the sample reporting an impairment using the 'Other impairment' category was low (4.4 per cent of the version 1 sample and 2.7 per cent of the version 2 sample). In version 1 , of those reporting an impairment using the 'Other impairment' category, two-fifths provided answers which were collapsible into an existing category; while in version 2, a quarter could be collapsed (Table 4.).

The most common conditions reported under the 'other impairment' category which were not obviously collapsible were circulatory disorders, gastrointestinal disorders and diabetes. With guidance it may be possible for interviewers to probe further; for example for those with diabetes interviewers could ask whether it affects their vision, and for circulatory disease whether it affects their stamina or breathing. Some respondents mentioned frequent tiredness connected with specific conditions such as diabetes. These respondents clearly did not connect their problem with a 'stamina' difficulty and the inclusion of the term 'fatigue' or 'tiredness' within the 'stamina or breathing' category may offer an additional opportunity to make the connection with their health condition and avoid using the 'other impairment' category for free text transcription.

A possible candidate for inclusion as a separate category was 'pain'; a number of respondents reported pain associated with their specific condition or conditions. Pain is often a symptom of a number of conditions, as well as connected with medication side effects and the effects of hospital investigations and procedures, and is relevant to activity restriction. 


\section{Table $4 \quad$ Other impairments which collapsed into an existing impairment category}

Version 1

$\%$ collapsible

\begin{tabular}{|c|c|}
\hline $\begin{array}{l}\text { Mobility/dexterity - } \\
\text { back problems; stretching problems; writing difficulty (use of hands); balance; bone problems; osteoporosis; limited joint } \\
\text { movements; arthritis; disc lesions; inability to maintain physical positions. }\end{array}$ & 19.1 \\
\hline $\begin{array}{l}\text { Stamina/breathing - } \\
\text { asthma; breathing problems; bronchitis. }\end{array}$ & 8.1 \\
\hline $\begin{array}{l}\text { Seeing - } \\
\text { eye condition; colour blindness. }\end{array}$ & 4.1 \\
\hline $\begin{array}{l}\text { Mental health - } \\
\text { depression; panic; alcoholism; social phobia; nervousness. }\end{array}$ & 5.9 \\
\hline $\begin{array}{l}\text { Learning/memory - } \\
\text { memory loss; learning difficulties; dyslexia. }\end{array}$ & 2.5 \\
\hline Total & 39.7 \\
\hline \multicolumn{2}{|l|}{ Version 2} \\
\hline $\begin{array}{l}\text { Mental health - } \\
\text { depression; alcohol abuse; anxiety: }\end{array}$ & 4.9 \\
\hline $\begin{array}{l}\text { Learning/memory - } \\
\text { short-term memory loss; learning difficulties; dementia, dyslexia; alzheimers: }\end{array}$ & 6.7 \\
\hline $\begin{array}{l}\text { Mobility/dexterity - } \\
\text { back problems; movement restrictions; arthritis; loss of grip; osteoporosis; un-operable hernia: }\end{array}$ & 13.8 \\
\hline $\begin{array}{l}\text { Social learning - } \\
\text { difficulties with social interaction }\end{array}$ & 1.0 \\
\hline Total & 26.4 \\
\hline
\end{tabular}

Source: Opinions 2009-10

Another weakness of the list relates to the combining of 'learning' and 'concentrating' with 'remembering'. There was evidence to suggest the 'learning' and 'concentrating' components dissuaded respondents with conditions affecting 'memory' from selecting this category. There was also evidence from consultation that the analysis of disaggregated categories to support policy would benefit from separating out 'memory' from 'learning' and 'concentrating' as the needs of these people are different.

\section{Correspondence between the impairment list and the FRS list of capacities}

The FRS disability module asks respondents about whether they have 'substantial' difficulty in a number of capacities such as mobility. There was interest among stakeholders in what level of correlation existed between the impairments contained in the field-test question and the FRS capacities, where similarity in categories existed. The OS also collected the FRS capacities for three of the five months of field testing allowing direct measurement at the individual level of the overlap in the reporting of impairments and capacities.

Table 5 shows the matrix of reporting of DDA capacities by the version 1 and version 2 impairment categories. 
Table 5 Percentage prevalence of impairment in those reporting
substantial difficulty in a specific capacity domain

\begin{tabular}{|c|c|c|c|c|c|c|c|c|}
\hline \multirow{2}{*}{ Capacity } & \multicolumn{8}{|c|}{ Version 1} \\
\hline & Seeing & Hearing & Mobility & Learning & $\begin{array}{l}\text { Mental } \\
\text { Health }\end{array}$ & Stamina & $\begin{array}{r}\text { Social } \\
\text { Learning }\end{array}$ & Other \\
\hline Moving about & 13.2 & 17.5 & 77.8 & 16.8 & 12.0 & 31.0 & 1.0 & 9.4 \\
\hline Balance/Coordination & 16.4 & 29.3 & 71.0 & 32.9 & 16.0 & 30.4 & 3.8 & 13.6 \\
\hline Lifting & 12.0 & 18.1 & 73.3 & 17.4 & 8.4 & 30.9 & 2.0 & 7.2 \\
\hline Manual dexterity & 19.0 & 24.4 & 86.5 & 28.1 & 17.3 & 41.4 & 2.7 & 10.1 \\
\hline Bladder/Bowel & 18.5 & 16.1 & 68.2 & 28.6 & 10.5 & 26.9 & 4.8 & 8.6 \\
\hline Communication & 33.1 & 42.3 & 51.6 & 25.7 & 14.7 & 29.6 & 2.6 & 9.1 \\
\hline Learn/Memory & 16.1 & 19.2 & 42.1 & 48.5 & 29.1 & 22.0 & 3.9 & 8.6 \\
\hline Danger & 38.7 & 26.6 & 54.2 & 30.0 & 44.1 & 32.7 & 8.2 & 9.6 \\
\hline Other & 12.6 & 19.6 & 40.9 & 23.4 & 23.2 & 33.7 & 3.6 & 31.4 \\
\hline \multicolumn{9}{|c|}{ Version 2 (percentage reporting capacity and impairment) } \\
\hline Capdcily & Seeing & Hearing & Mobility & Learning & $\begin{array}{l}\text { Mental } \\
\text { Health }\end{array}$ & Stamina & $\begin{array}{r}\text { Social } \\
\text { Learning }\end{array}$ & Other \\
\hline Moving about & 12.7 & 16.9 & 89.5 & 18.5 & 11.4 & 34.3 & 1.3 & 5.2 \\
\hline Balance/Coordination & 16.0 & 22.8 & 71.7 & 35.3 & 16.7 & 32.2 & 6.1 & 6.8 \\
\hline Lifting & 10.3 & 19.3 & 72.0 & 15.2 & 7.3 & 32.2 & 1.2 & 4.4 \\
\hline Manual dexterity & 13.7 & 20.4 & 76.8 & 26.9 & 20.2 & 36.2 & 3.3 & 8.8 \\
\hline Bladder/Bowel & 9.3 & 17.6 & 56.4 & 17.0 & 10.6 & 26.1 & 0.0 & 7.9 \\
\hline Communication & 34.7 & 52.1 & 42.7 & 34.1 & 16.2 & 20.9 & 6.2 & 7.3 \\
\hline Learn/Memory & 13.8 & 16.7 & 49.4 & 65.3 & 30.2 & 30.2 & 7.1 & 9.2 \\
\hline Danger & 20.1 & 15.3 & 50.4 & 55.9 & 50.3 & 36.7 & 13.9 & 0.0 \\
\hline Other & 19.1 & 17.4 & 33.3 & 19.7 & 34.5 & 37.3 & 5.1 & 11.8 \\
\hline
\end{tabular}

Source: ONS Opinions Survey 2009-10

A compelling level of correspondence is suggested in this matrix between some capacities and impairments. The reporting of 'mobility' impairment has a strong overlap with the capacities 'moving', 'balance', 'lifting' 'manual dexterity' and 'continence'. Another important overlap is present between the capacity 'learning' and the impairment 'learning or concentrating or remembering'.

\section{Conclusions of stage 1 field test}

The question capturing persistent physical or mental health conditions or illnesses should contain a 12-month time reference encompassing the past and future 12 months to improve the time frame comparability with the current FRS and LOS questions. The exclusion of the terms 'disability' and 'impairment' should continue as the evidence suggests prevalence estimates are insufficiently discrepant with other sources by their exclusion, and the application of the disablement process and the International Classification of Functioning, Disability and Health (WHO, 2001) advocates 
the separation of concepts of illness, functional impairment and disability (that is activity and participation restriction).

The impairment list should route from the question capturing persistent physical or mental health conditions or illnesses; the added benefit of capturing impairment for the whole sample is unsubstantiated and adds to interview length and costs.

Some avoidance of the use of the 'other impairment' category is possible through the provision of interviewer instructions and guidance on probing. For example, probing the respondent to consider whether 'stamina or breathing' is affected by his or her heart condition.

The merits of including the term 'fatigue' in the 'stamina or breathing' category and a separate category eliciting 'pain' should be judged following any further evidence supporting their inclusion from the stage 2 field test results.

To improve policy relevance, the category 'learning or concentrating or remembering' requires amendment; specifically creating new categories of 'memory impairment' and 'learning or concentrating or understanding impairment'.

The measurement of activity restriction can be captured effectively using the terms difficulty and the plain English extent categories of 'a little' and 'a lot'. There was an absence of evidence supporting the capture of frequency of restriction to offset any misclassification of those with intermittent activity restriction. The duration of activity restriction should have categories enabling breakdowns to meet alternative legislation guidelines set nationally and in Europe.

\section{Field Testing Stage 2}

\section{Introduction}

The second stage of field testing was conducted to further test the performance of the questions in measuring the population prevalence of Equality Act (National Archive, 2010) disability and the relationship the field-test questions have with the question on disability included in the decennial 2011 Census of population.

\section{Two question suites were tested (see Annex D):}

1. the proposed harmonised disability question suite, amended to take account of stage 1 field test findings

2. the Scottish 2011 Census question on health conditions and the 2011 Census question on disability asked across the UK constituent countries

The specific purposes for this stage of field testing are to:

a) compare the prevalence of core disability, as measured by the FRS with the proposed harmonised disability question suite

b) assess the level of agreement in prevalence between the harmonised suite's derivation of disability by extent and that derived from the 2011 Census question on disability

c) assess the scope to deliver Equality Act and EU-SILC outputs with and without the duration filter 


\section{Methods}

\section{Data source and study population}

The ONS Opinions Survey provided the data collection vehicle to test these questions during the months of May, June and July 2010. The estimates derived were compared with other sources collecting similar data for external validation. These included:
a) Family Resources Survey
b) Life Opportunities Survey
c) General Lifestyle Survey

The Opinions survey sample was split into two equal parts; one half of the sample were given the proposed harmonised questions, while the other half were given the questions planned for the decennial 2011 Census of population. The sample sizes were 1,726 for the proposed harmonised question suite and 1,768 for the census questions.

\section{Question Suite Versions}

The proposed harmonised suite included questions determining:

a) the presence of a physical or mental health condition or illness

b) impairment status, by type

c) extent of difficulty carrying out day-to-day activities, by duration

A number of changes to the proposed harmonised questions were introduced in stage 2. First, the time frame in the question eliciting the presence of a persistent health condition or illness is more inclusive, referring to both the past 12 months and also an expectation of duration for a further 12 months. Second, the impairment list has separated out the terms 'memory' from 'learning, understanding or concentrating' and replaces the term 'social learning difficulty' (referring to autistic spectrum conditions) with 'socially or behaviourally'. Third, the question eliciting activity restriction includes the term 'reduce your ability to carry out day-to-day activities' with running prompt plain English response categories 'yes, a lot', 'yes, a little', or 'not at all'. Fourth, a question measuring frequency of activity restriction is excluded. 


\section{Scope of testing elements}

A number of criteria were set to determine the fitness for purpose of these questions:

a) Is the prevalence of a physical or mental health condition lasting or expected to last 12 months or more consistent with estimates derived from the equivalent questions asked on the FRS 2008-09, the LOS 2009-10 and the GLF 2008 and 2009?

b) How does prevalence of impairment compare with LOS published estimates (ONS, 2009)?

c) Is there evidence that estimates of activity restriction fall from the stage 1 level by excluding a question on frequency?

d) Is the estimate of core Equality Act disability prevalence, derived from the proposed harmonised questions, comparable with that derived from the proposed 2011 Census question on disability and with external source estimates derived from the FRS 2008-09 and 2009-10 and LOS 2009-10 questions

e) Is the estimate of persistent illness and disability derived from the proposed harmonised questions plausible for EU-SILC data requirements based on historical estimates published on the Eurostat website? Does the duration filter improve coherence?

f) Is there evidence that the capture of extent of activity restriction inflates prevalence compared with sources using dichotomised 'Yes' and 'No' response choices?

\section{Results and recommendations}

\section{Physical and mental health conditions}

Table 6 compares the prevalence of a health condition or illness measured using the field test stage 2 question with estimates derived from the FRS 2008-09 and 2009-10, the LOS 2009-10 and the GLF 2008 and 2009 data.

For persons, the stage 2 field test prevalence was 5.4 percentage points higher than the version 2 stage 1 field test prevalence. The stage 2 prevalence is also somewhat higher than the equivalent question asked on the FRS using both 2008-09 and 2009-10 data; however, it is lower than both the LOS 2009-10 and the GLF 2008 and 2009 estimates. The field test question's estimate across ages and by sex are more consistent with LOS and the GLF than with the estimates derived from the FRS. 


\section{Table 6 Percentage prevalence of a physical or mental health condition: by source}

Great Britain

\begin{tabular}{lrrrrrr}
\hline Parameter & Field test & LOS 09-10 & FRS 08-09 & FRS 09-10 & GLF 2008 & GLF 2009 \\
\hline Persons & 33.7 & 35.1 & 29.2 & 29.6 & 34.1 & 35.2 \\
Men & 31.5 & 34.0 & 28.2 & 28.8 & 33.6 & 35.0 \\
Women & 35.7 & 36.1 & 30.2 & 30.3 & 34.6 & 35.5 \\
Persons 16-24: & 15.9 & 12.5 & 10.4 & 10.7 & 13.0 & 12.5 \\
Persons 25-64: & 28.4 & 30.9 & 24.2 & 24.5 & 28.1 & 29.4 \\
Persons 65 and over: & 64.6 & 63.6 & 61.4 & 61.1 & 60.5 & 61.6 \\
\hline
\end{tabular}

While the exclusion of the terms 'disability' and 'impairment' from the body of the field tested question may have a marginal effect on suppressing prevalence, the proposed harmonised question has the advantage of disentangling concepts of health conditions or illnesses and disability, and is therefore more specific in what it is measuring; that is the prevalence of any physical or mental health conditions or illnesses. Questions which mix these concepts leave uncertainty about the distribution between these states and they carry the potential inconsistency of classifying disability in the absence of activity restriction, which runs counter to the harmonised definition agreed and presented in the accompanying article in this issue of Health Statistics Quarterly (White, 2011), and the EU-SILC guideline of separating out the measurement of chronic illness from disability.

For these reasons the sub-group agreed the harmonised standard question should:

1. exclude the terms 'disability' and 'impairment'

2. adopt a 12-month or more time frame taking account of the past duration and expected future duration

\section{Prevalence of impairment}

The question on impairment was given only to those reporting a physical or mental health condition in the previous question in stage 2 testing. The prevalence of impairment is shown in table 7 together with the LOS impairment prevalence (the LOS collected data on a wider range of impairments and therefore for some impairments, no equivalent figure is available from the field tested question).

The most common impairment using the field test question was 'mobility' at 16.6 per cent, encompassing difficulty climbing stairs, walking short distances and lifting and carrying objects; 'stamina and breathing' impairment affected 9.0 per cent of the population. The prevalence estimates are higher in each case across all impairment categories than that found in stage 1 testing.

The prevalence of impairment overall using the field-test question was 4.1 percentage points lower than that found in LOS, but this does not compare like with like as LOS collects a wider range of impairments across the whole sample which may affect the overall prevalence estimate. 


\section{Table $7 \quad$ Percentage prevalence of impairment: by source, persons aged 16 years and over}

\begin{tabular}{|c|c|c|}
\hline Impairment & Field Test & LOS \\
\hline Vision & 4.0 & 3.0 \\
\hline Hearing & 4.1 & 3.0 \\
\hline Speaking & * & 1.0 \\
\hline Mobility(encompasses dexterity in field test question) & 16.6 & 8.0 \\
\hline Dexterity & * & 6.0 \\
\hline Long-term pain & * & 18.0 \\
\hline Learning & 2.7 & 2.0 \\
\hline Intellectual & * & 0.0 \\
\hline Memory & 2.9 & 3.0 \\
\hline Mental Health & 3.9 & 4.0 \\
\hline Stamina or Breathing (breathing only in LOS) & 9.0 & 3.0 \\
\hline Behavioural (e.g. autistic spectrum conditions) & 0.7 & 1.0 \\
\hline Chronic health conditions & * & 12.0 \\
\hline Other & 3.5 & 1.0 \\
\hline Any Impairment & 25.9 & 29.0 \\
\hline
\end{tabular}

Source: ONS Opinions Survey 2010 and Life Opportunities Survey 2009-10

The prevalence of sight and hearing impairment is approximately 1 percentage point lower in LOS, while a good level of equivalence is found for 'learning', 'memory', 'mental health' and 'behavioural' impairment. Mobility impairment is not consistent with LOS; however, as LOS separates out 'dexterity' from 'mobility' and the proposed harmonised question combines them, the aggregated LOS 'mobility' and 'dexterity' prevalence estimate improves consistency with the field-test question.

The routeing approach of the harmonised question produces a good level of consistency with the non-routed LOS impairment categories, suggesting reliable prevalence estimates are achievable using routeing. Consequently, the sub-group recommended only those with a health condition or illness are asked about type of impairment.

However two changes to the list were recommended following field test findings: These were:

a) adding the term 'fatigue' to the 'stamina and breathing' category, to take account of responses citing tiredness as a consequence of their health condition or illness

b) a mobility-specific category, encompassing an ability to walk short distances and climb stairs; and a 'dexterity' category, with examples of moving and carrying objects and using work related devices such as a keyboard

The latter change improves consistency with the LOS and takes account of the different policy needs presented by mobility and dexterity impairment for care and fitness for work 


\section{Effect of excluding 'frequency' on estimates of activity restriction}

The proposed questions produce estimates of activity restriction of 21.9 per cent, exceeding those estimates captured at stage 1 where a question on frequency was asked. Consequently, the risk of misclassifying people with intermittent restrictions by excluding a question capturing 'frequency' of activity restriction is unlikely, suggesting activity restriction can be captured adequately using a question capturing extent of restriction. Therefore the sub-group recommended the exclusion of a question capturing frequency, thereby saving interview time.

\section{Comparability in estimates of disability}

There was a need to test how comparable estimates of disability using the proposed harmonised questions are with those likely from the decennial 2011 Census of population and estimates derived form other social survey data sources. Table 8 compares estimates derived from the proposed harmonised questions, the 2011 Census question, and external social survey sources.

There is a good level of agreement in estimates derived from the proposed harmonised questions and the 2011 Census question both overall and by extent of disability. Both questions measure disability at 21.9 per cent of the population aged 16 years and over; the harmonised question's higher category of 'a lot' is estimated at slightly more than 10 per cent, which exceeds the GLF estimate of 8.7 per cent prevalence of 'strongly limited' in 2009; but overall disability coheres well with the GLF 2009 estimates. The prevalence of core disability measured in the FRS 2008-09 data is 21.4 per cent, and in the FRS 2009-10 data is 21.3 per cent, which also compares favourably with the proposed harmonised question; however, the LOS wave 1 interim estimate is 4 percentage points higher.

The comparability in prevalence with the 2011 Census question and with the FRS and GLF estimates, despite wording and routeing differences, suggests the harmonised questions will provide the functionality to measure inter-censal prevalence of disability from a census benchmark in 2011, and not risk a radical discontinuity to FRS or GLF estimates. 


\section{Percentage prevalence of activity restriction by extent, persons aged 16 years and over}

Source

Percentage

Field test question - Opinions 2010

Does your condition or illness/do any of your conditions or illnesses reduce your ability to carry out day-to-day activities

'Yes a lot' or 'Yes a little'?

'Yes, a lot'

'Yes, a little'

FRS question - 2008-09

Does this/do these health problems or disabilities mean that you have substantial difficulties with any of these areas of your life? Mobility; lifting; manual dexterity; continence; communication; memory, concentrate, understand or learn; physical danger; balance; other

FRS question - 2009-10

Does this/do these health problems or disabilities mean that you have substantial difficulties with any of these areas of your life? Mobility; lifting; manual dexterity; continence; communication; memory, concentrate, understand or learn; physical danger; balance; other

\section{LOS question 2009-10 interim results}

Does this/do these health problems or disabilities mean that you have substantial difficulties with any of these areas of your life? Mobility; lifting; manual dexterity; continence; communication; memory, concentrate, understand or learn; physical danger; balance; other

GLF question 2009

Does this illness(es) or disability(ies) limit your activities in any way?

Limited or strongly limited

Strongly limited

Field Test of 2011 Census question - Opinions 2010

Are your day-to-day activities limited because of a health problem or disability which has lasted or is expected to last 12 months or more?

Yes, limited a lot or yes limited a little

Yes, limited a lot

The prevalence of disability derived using the field-tested question's terminology of 'reduced ability carrying out day-to-day activities' and the plain English response categories coheres well with estimates from other sources and the 2011 Census question, suggesting the plain English response options are interpreted by respondents in a similar way to terms such as 'substantial difficulty' and 'limited activities'.

\section{Plausibility for EU-SILC estimates of persistent illness and disability}

The EU-SILC variable guideline requires capturing data on limitation carrying out daily activities people usually do for the past six months or more by extent of limitation. Consequently, the EUSILC classification for disability requires the application of the duration question filter to ensure the past six months or more criterion is complied with.

Table 9 shows the estimates for EU-SILC disability derived from the proposed harmonised questions with the EU-SILC estimates available from the EU-SILC health variables data cube on the Eurostat website (Eurostat a, 2011). 


\section{EU-SILC derived disability percentage prevalence, persons aged 16 years and over}

\begin{tabular}{|c|c|c|}
\hline Source & Percentage & $\begin{array}{r}95 \% \text { confidence } \\
\text { limits }\end{array}$ \\
\hline $\begin{array}{l}\text { Field test question - Opinions } 2010 \\
\text { For how long has your ability to carry out day-to-day activities been reduced? } \\
\text { Less than six months or between } 6 \text { and } 12 \text { months or } 12 \text { months or more }\end{array}$ & 21.9 & $20.0-23.9$ \\
\hline Less than six months & 1.3 & $0.7-1.8$ \\
\hline Between 6 months and 12 months & 1.8 & $1.2-2.5$ \\
\hline 12 months or more & 18.8 & $17.0-20.7$ \\
\hline Revised EU-SILC estimate taking account of duration & 20.7 & $18.8-22.6$ \\
\hline $\begin{array}{l}\text { Revised EU-SILC estimate of 'Severely hampered in daily activities' using the activity restriction } \\
\text { category 'Yes, a Lot' and the duration categories 'between six months and } 12 \text { months' and '12 } \\
\text { months or more' }\end{array}$ & 9.9 & $8.5-11.3$ \\
\hline $\begin{array}{l}\text { Revised EU-SILC estimate of 'Hampered in daily activities to some extent' using the activity } \\
\text { restriction category 'Yes, a little' and the duration categories 'between six months and } 12 \text { months' } \\
\text { and ' } 12 \text { months or more' }\end{array}$ & 10.8 & $9.3-12.2$ \\
\hline $\begin{array}{l}\text { EU-SILC } 2009 \text { estimate on Eurostat website - conflated categories 'Severely hampered in daily } \\
\text { activities' and 'Hampered in daily activities to some extent' }\end{array}$ & 20.8 & \\
\hline EU-SILC 2009 estimate on Eurostat website - 'Severely hampered in daily activities' & 9.1 & \\
\hline EU-SILC 2009 estimate on Eurostat website - 'Hampered in daily activities to some extent' & 11.7 & \\
\hline
\end{tabular}

Sources: ONS Opinions Survey 2010; Eurostat

The harmonised questions have a satisfactory level of consistency with the EU-SILC estimates recorded on the Eurostat website, both overall and separated out into extent categories. This represents reassuring evidence that the proposed harmonised questions not only provide a closer affinity with the EU-SILC target variable concept and guidelines, but will also deliver an acceptable level of continuity with previous activity limitation estimates.

The prevalence of long-standing illness in 2009 for the UK published on the Eurostat website (Eurostat b, 2011) for persons aged 16 years and over was 33.6 per cent. The prevalence of a physical or mental health condition or illness lasting or expected to last 12 months or more derived from the proposed harmonised question tested at stage 2 delivered a prevalence of 33.7 per cent. The proximity of these estimates suggest the proposed harmonised question wording and time frame used will not risk a serious discontinuity in the EU-SILC time series of long-standing illness, and has the added benefit of greater compliance with the EU-SILC target variable concept and guidelines for the collection of long-standing illness.

The sub-group members agreed the proposed question inputs for EU-SILC data collections would fulfil the requirements under the EU regulation. 


\section{Inflation in prevalence by capturing extent of activity restriction}

The prevalence of activity restriction estimates reported in table 8 suggest the capture of extent will not lead to an artefactual increase in the prevalence of activity restriction compared with questions using dichotomised response categories of 'Yes' or 'No', (that is, the possible risk that survey respondents perceive the threshold for reporting restriction to be lower using the category ' $Y e s, a$ little'. The perceived risk of a greater number of people willing to use the 'Yes a little' category in the proposed harmonised question and the category 'Yes, limited a little' in the 2011 Census, but reluctant to use a 'Yes' response when asked whether their activities were 'limited' in a 'Yes' or 'No' response option was not borne out in these results.

\section{Conclusions of stage 2 field test}

The results from the second stage of field testing suggests the absence of the term 'disability' from the body of the proposed harmonised question will not introduce a serious discontinuity in the prevalence estimates following implementation in social survey sources. The added value of specificity in what is measured and the avoidance of mixed concepts in the same question produces greater alignment with the conceptual frameworks of disability stipulated in national legislation, European regulation and the internationally recognised ICFDH.

The capture of type of impairment requires two amendments to the list; specifically, the addition of the term 'fatigue' to the 'stamina and breathing' category, and the addition of a 'dexterity' category to take account of the different policy needs presented by people with dexterity impairment as opposed to those with mobility impairment.

The routeing of the impairment question from the question eliciting a physical or mental health condition or illness appears justified as prevalence estimates are similar to the estimates achieved in LOS. However, government stakeholders asked for an acknowledgement in the harmonised standard to allow administration of the impairment question to the whole sample for those surveys wanting to capture impairment type among the general population - whether a respondent reported a physical or mental health condition or illness or not. The sub-group agreed to this proviso and an alternative version of the question for general sample administration was developed and is shown under the heading 'data inputs' below.

The proximity between the estimate of core DDA disability derived from the criterion (standard on which a judgement is made) 'a substantial difficulty in one or more capacity domains' used in FRS publications and the estimates from both the field test question's combined categories of ' $Y$ es, a lot' or 'Yes, a little' reduced ability to carry out day-to-day activities and the 2011 Census combined categories 'Yes, limited a lot' and 'Yes, limited a little' suggests the term 'substantial difficulty' in at least one capacity domain agrees with the combined responses of 'Yes, a lot' and 'Yes, a little'.

The field test results show that the effect of using a duration filter (that is. a question determining how long a person has had activity restriction) to classify a case of disability will reduce prevalence estimates marginally; a 12 months or more time filter reduces the prevalence of activity restriction to 18.8 per cent, while a 6 months or more time filter reduces prevalence to 20.7 per cent.

Consequently, the application of the duration filter is dubious for both Equality Act estimates of the core currently disabled population or for benchmarking with the 2011 Census disability estimate. 
However, the sub-group members agreed the inclusion of the duration filter question as tested should be retained in the primary harmonised standard to provide data users with the agility to either exclude or include depending on the purpose of the analysis.

The application of the duration filter improves comparability with the EU-SILC 2009 estimate; the estimate of 'Yes, a lot' of reduced ability carrying out day-to-day activities in question 2a and filtering using the duration categories 'between 6 months and 12 months' and ' 12 months or more' is 9.9 per cent which compares favourably with the EU-SILC 2009 estimate of 'severely hampered in daily activities' of 9.1 per cent, while the estimate of 'Yes, a little' reduced ability carrying out day-to-day activities and the same duration categories is 11.0 per cent, which also compares favourably with the EU-SILC 2009 estimate of 'hampered to some extent' of 11.8 per cent. These estimates have a satisfactory level of coherence with the 2009 UK estimates published on the Eurostat website (Eurostat, 2011).

\section{Harmonised standard disability data inputs and statistical measures}

The following question inputs were agreed as the primary harmonised standard questions to provide derivations of statistical measures of persistent illness and disability for implementation in household social surveys using an in-person interview mode of data collection (Box 1). They have been scrutinised by the NSHG, the GSS SPSC and the Heads of Profession.

These questions are designed to derive the following classifications of persistent health conditions and illnesses and disability for Equality Act 2010 monitoring and EU-SILC data collections (Box 2). 


\section{Box 1 Primary harmonised questions}

\section{Question 1a:}

To establish whether a person has a physical or mental health condition or illness

Ask all if $>=16$ years of age, ask by proxy if $<16$ or not fit to respond in person.

Do you have any physical or mental health conditions or illnesses lasting or expected to last 12 months or more?

1. Yes;

2. No

Spontaneous only:

3. Don't know;

4. Refusal

\section{Question 1b(i) (standard version):}

The effect of any physical or mental health conditions or illnesses on functioning Ask if $1 a=$ Yes.

Do any of these conditions or illnesses affect you in any of the following areas?

Show card and code all that apply:

1. Vision (for example blindness or partial sight)

2. Hearing (for example deafness or partial hearing)

3. Mobility (for example walking short distances or climbing stairs)

4. Dexterity (for example lifting and carrying objects, using a keyboard)

5. Learning or understanding or concentrating

6. Memory

7. Mental health

8. Stamina or breathing or fatigue

9. Socially or behaviourally (for example associated with Asperger's syndrome and other autistic spectrum conditions)

10. Other (please specify)

Spontaneous only:

11. None of the above

12. Refusal 


\section{Box 1 Primary harmonised questions}

Ask if $Q 1 b=10$

Please specify how your condition(s) or illness(es) affect you. (STRING 255)

Question 1b(ii) (optional version):

Whether any physical or mental health condition or illness affects functioning, irrespective of whether a positive answer was given to Question 1a

Ask All.

Do you have any health conditions or illnesses which affect you in any of the following areas?

Continue as for Question $1 \mathrm{~b}(\mathrm{i})$

Question 2a:

Whether a person with any physical or mental health conditions or illnesses have activity restriction

Ask if $1 \mathrm{a}=$ Yes.

Does your condition or illness/do any of your conditions or illnesses reduce your ability to carry out dayto-day activities?

Running prompt:

1. Yes, a lot

2. Yes, a little

3. Not at all

Question 2b:

For what period of time has the person experienced activity restriction.

Ask if $2 a=$ Yes, $a$ lot or Yes, a little.

For how long has your ability to carry out day-to-day activities been reduced?

Running prompt:

1. Less than six months

2. Between six months and 12 months

3. 12 months or more 


\section{Box 2 Key classifications based on the primary harmonised questions}

Classification of disability for the core population with rights under the Equalit $y$ Act

Question 1a $=$ Yes

AND

Question 2a = Yes, a little OR Yes, a lot

\section{EU-SILC long-standing health condition or illness}

Question $1 \mathrm{a}=$ Yes

\section{EU-SILC classification of disability by extent}

Estimate of severely hampered in daily activities

Question 1a $=$ Yes

AND

Question $2 \mathrm{a}=$ Yes, a lot

AND

Question $2 \mathrm{~b}=$ Between six months and 12 months OR 12 months or more

Estimate of hampered in daily activities to some extent

Question 1a $=$ Yes

AND

Question 2a = Yes, a little

AND

Question $2 b=$ Between six months and 12 months OR 12 months or more 
Further documentation to aid implementation, including those existing questions the primary harmonised standard questions will replace, interviewer instructions, output categories, the timetable for implementation in ONS social survey sources and the future reporting of survey estimates will be made available on the ONS harmonisation website during the course of 2011.

\section{Next Steps}

The primary harmonised disability questions are planned for implementation on the Integrated Household Survey (IHS) core module in January 2012, replacing the traditional two questions on long-standing illness and activity restrictions. This will facilitate the derivation of measures of disability using the new harmonised question inputs and classification at national and sub-national level, which can be updated annually in IHS statistical bulletins. Annual estimates of disability-free life expectancy at sub-national level will also be feasible using prevalence data from this data source in future years, enabling the years of life expected to be spent free from disability to be regularly and reliably updated for Unitary Authorities and Local Authority Districts.

The capture of chronic illness and activity restriction data for EU-SILC data collection is expected to transfer from the GLF to the FRS in April 2012: this will provide the data inputs for Eurostat to construct measures of disability for the UK, based on the harmonised standard questions, and UK official measures of the core currently disabled population with rights under the Equality Act used in FRS publications.

More literature pertaining to the revised harmonised questions, including the cognitive testing and field testing reports and a guide for implementation on surveys using alternative data collection modes, such as postal surveys, will be uploaded onto the National Statistics Harmonisation Group website during 2012.

Further work on harmonisation planned for 2011-12 are to:

a) assess the need to adapt the questions using alternative modes of data capture such as postal and online surveys

b) conduct further consultation on the development of a secondary harmonised standard to capture the social barriers to participation in key areas of life and the functionality of data inputs to derive measures of participation restriction and the disproportionate impacts of social barriers on people with impairments relative to people without impairment

c) carry out a fact finding exercise on disability data collections in administrative sources with a view to improving the harmonization of this data and its fitness for use to derive statistical measures of disability to support planning

\section{Conclusion}

This article has updated developments in the harmonisation of disability data collection to provide greater consistency in the statistical measures of disability from national household social surveys using a face-to-face mode of data collection. The primary harmonized standards have a stronger foundation in an internationally recognized framework of disability and in the World Health Organisation's International Classification of Functioning, Disability and Health. 
The field testing of these harmonised standard questions has provided evidence of their fitness for use in social surveys to deliver the outputs necessary to meet the legislative criteria specifying the core currently disabled population with rights under the Equality Act 2010, the data required by Eurostat to compare disability and derive the Healthy Life Years Structural Indicator across member states of the European Union, and have affinity with the estimates of disability expected from the 2011 Census.

The improvement in consistency expected through harmonisation and the stronger anchoring of these questions to an internationally recognised conceptual framework of disability will improve the relevance of the statistical measures of disability derived for monitoring the inequality experienced by people with impairment, setting outcome measures to mitigate these inequalities and support wider service needs assessment. 


\section{References}

Eurostat a (2011). Self-perceived limitations in daily activities. Available on the Eurostat website at: www.appsso.eurostat.ec.europa.eu/nui/show.do?dataset=hlth silc 12\&lang=en

Eurostat b (2011). People having a long-standing illness or health problem. Available on the Eurostat website at:

www.appsso.eurostat.ec.europa.eu/nui/show.do?dataset=hlth silc 04\&lang=en

National Archive (1995). Disability Discrimination Act 1995. Available from the national archive website at: www.legislation.gov.uk/ukpga/1995/50/contents

National Archives (2010). Equality Act. Available at the National Archives website: www.legislation.gov.uk/ukpga/2010/15/contents

Office for National Statistics (2007) Report from the review of equality data. Available on the Office for National Statistics website at:

www.statistics.gov.uk/downloads/theme social/EDR Final.doc.pdf

ONS Opinions Survey (2011). Further information available on the ONS website at:

www.ons.gov.uk/about/who-we-are/our-services/omnibus-survey/index.html

ONS (2010). Life Opportunities Survey Interim Results 2009-10. OPSI Richmond. Available on the ONS website at: www.statistics.gov.uk/about/Methodology by theme/downloads/los-interimresults-09-10.pdf

White, C. (2011). Update on the harmonisation of disability data collection in UK surveys (Part 1) Health Statistics Quarterly, Autumn 2011. Available on the Office for National Statistics website at: www.statistics.gov.uk/CCl/article.asp?ID $=2728$

World Health Organisation (2001). International Classification of Functioning, Disability and Health. Available on the World Health Organisation's website at: www.who.int/classifications/icf/en/ 


\section{Annex A}

Field Test Stage 1 Question Suites: following cognitive testing and consultation findings

Version 1 questions used in half the ONS Opinions Survey sample (October 2009 - February 2010)

Introductory statement

The following questions are asked on behalf of the Office for National Statistics. They ask you about any health conditions you have which are long-lasting in nature and any problems you have with normal daily activities.

ASK ALL

Q1a. Do you have any physical or mental health conditions or illnesses which have lasted for 12 months or more?

1. Yes

2. No

Spontaneous only

3. Don't Know

4. Refuse

ASK IF Q1a=1 and code all that apply

Q1b. Do you have difficulty in any of the following areas because of your health condition(s) or illnesses?

Show card and code all that apply

1. Seeing (due to blindness or partial sight).

2. Hearing (due to deafness of partial hearing).

3. Mobility, such as difficulty walking short distances, climbing stairs, or lifting and carrying objects.

4. Learning or concentrating or remembering.

5. Mental Health

6. Stamina or breathing difficulty

7. Social Learning difficulty (for example, due to Autistic Spectrum Disorder or Asperger's)

8. Other (please state).

9. None of the above

$A S K I F Q 1 b=8$

Please specify the other area(s) in which you have difficulty? 


\section{ASK IF Q1a=1}

Q2a. For at least the past 12 months, have you had difficulty in carrying out day-to-day activities (in the home, at work or during leisure time)?

\section{Running prompt}

1. Always

2. Most of the time

3. Sometimes

4. Not at all

\section{ASK IF Q2a IS LESS THAN 4}

Q2b. To what extent do you have difficulty carrying out day-to-day activities?

Running prompt

1. A little difficulty $O R$

2. A lot of difficulty 
Version 2 questions used in half the Opinions Survey sample (October 2009 - February 2010)

Introductory statement

The following questions are asked on behalf of the Office for National Statistics. They ask you about any problems you have with normal daily activities and any health conditions you have which are long-lasting in nature.

\section{ASK ALL}

Q2a. Do you have any physical or mental health condition(s) or illnesses that are expected to last 12 months or more?

Running prompt

1. Yes

2. No

Spontaneous only

3. Don't Know

4. Refuse

\section{ASK ALL}

Q2b. Do you have difficulty in any of the following areas because of a health condition(s) or illnesses?

\section{Show card}

1. Seeing (due to blindness or partial sight)

2. Hearing (due to deafness of partial hearing)

3. Mobility, such as difficulty walking short distances, climbing stairs, lifting \& carrying objects.

4. Learning or concentrating or remembering.

5. Mental Health

6. Stamina or breathing difficulty

7. Social Learning difficulty (for example, due to Autistic Spectrum Disorder or Asperger's')

8. Other (please state).

9. None of the above.

ASK IF Q2b = 8

Please specify the other area(s) in which you have difficulty? 


\section{ASK IF Q2a=1 or Q2b LESS THAN 9}

Q3a In relation to your condition(s) or illnesses, to what extent do you have difficulty carrying out day to day activities?

\section{Running prompt}

1. A lot of difficulty

2. A little difficulty

3. No difficulty

\section{ASK IF Q3a=1 or 2}

Q3b. For how long have you had difficulty with day-to-day activities?

\section{Running prompt}

1. Less than 6 months

2. Between 6 \& 12 months

3. 12 months or more 


\section{Annex B}

\section{Family Resources Survey Disability Module (2008-09)}

FRS0805B.BU.QHealth1.Adult.Health

Do you have any long-standing illness, disability or infirmity? By 'long-standing' I mean anything that has troubled you over a period of at least 12 months or that is likely to affect you over a period of at least 12 months.
(1) Yes
(2) No

ASK IF Health = YES:

FRS0805B.BU.QHealth1.Adult.HProb

Does this physical or mental illness or disability (Do any of these physical or mental illnesses or disabilities) limit your activities in any way?
(1) Yes
(2) No

ASK IF Health = YES:

\section{FRS0805B.BU.QHealth1.Adult.DisDif}

Does this/Do these health problem(s) or disability(ies) mean that you have substantial difficulties with any of these areas of your life?

(1) Moving: Mobility (moving about)

(2) Lifting: Lifting, carrying or moving objects

(3) Hands: Manual dexterity (using your hands to carry out everyday tasks)

(4) Bladder: Continence (bladder and bowel control)

(5) Speech: Communication (speech, hearing or eyesight)

(6) Learn: Memory or ability to concentrate, learn or understand

(7) Danger: Recognising when you are in physical danger

(8) Balance: Your physical co-ordination (eg: balance)

(9) Other: health problem or disability

(10) None: None of these 


\section{Annex C}

General Lifestyle Survey questions on long-standing illness and activity limitation

Ask all

\section{LSIII}

Do you/does (..) have any long-standing illness, disability or infirmity - by long-standing I mean anything that has troubled you over a period of time or that is likely to affect you/him/her over a period of time?

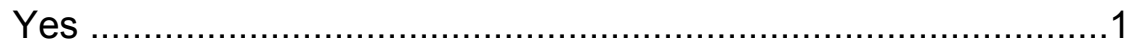

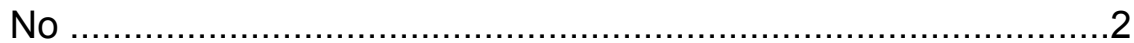

\section{Ask if $L S I I I=1$}

\section{IIILim}

Does this illness or disability (Do any of these illnesses or disabilities) limit your/his/her activities in any way?

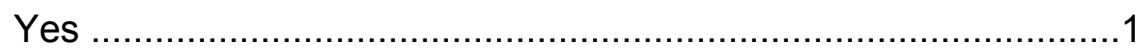

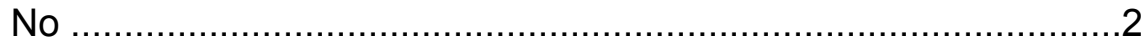




\section{Annex D}

\section{Proposed harmonised question suite following stage 1 field test and further consultation}

Sample 1 ONS Opinions Survey (May 2010 - July 2010)

Introduction

These questions are asked on behalf of the Office for National Statistics. They ask you about any health conditions you have which are long-lasting in nature, the effect of any health conditions in your functioning and any problems you have with normal day-to-day activities.

\section{ASK ALL}

Q1a. Do you have any physical or mental health conditions or illnesses lasting or expected to last 12 months or more?

1. Yes

2. No

Spontaneous only

\section{Don't Know}

4. Refuse

\section{ASK IF Q1a = 1}

Q1b. Does this condition or illness/do these conditions or illnesses affect you in any of the following areas?

Show Card code all that apply

1. Vision (for example, blindness or partial sight)

2. Hearing (for example, deafness or partial hearing)

3. Mobility (for example, walking short distances or climbing stairs or lifting/carrying objects)

4. Learning or understanding or concentrating

5. Memor y

6. Mental Health

7. Stamina or Breathing

8. Socially or Behaviourally (for example, Autism or Asperger's Syndrome)

9. Other, please specify

10. None of the above (Spontaneous only)

11. Don't Know (Spontaneous only)

12. Refusal (Spontaneous only)

\section{ASK IF Q1b $=9$}


Please specify how your condition(s) affect you. (String 255)

ASK IF Q1a $=1$

Q2a. Does your condition or illness/do any of your conditions or illnesses reduce your ability to carry out day-to-day activities?

\section{Running prompt}

1. Yes, a lot

2. Yes, a little

3. Not at all

ASK IF Q2a $=1$ or 2

Q2b. For how long has your ability to carry out day-to-day activities been reduced?

Running prompt

1. Less than six months

2. Between six months and 12 months

3. 12 months or more 


\section{Scottish Census 2011 Health Conditions and UK Census 2011 Disability Questions}

Sample 2 ONS Opinions Survey (May 2010 - July 2010)

Introduction

These questions ask you about any long-standing health conditions you may have and any problems you have with day-to-day activities.

\section{ASK ALL}

Q1. Do you have any of the following conditions which have lasted or are expected to last at least 12 months?

1. Deafness or partial hearing loss

2. Blindness or partial sight loss

3. A learning disability (for example, Down's syndrome)

4. A learning difficulty (for example, dyslexia)

5. A developmental disorder (for example, autistic spectrum disorder or Asperger's syndrome)

6. A physical disability

7. A mental health condition

8. A long-term illness, disease or condition

9. Any other condition (please specify)

10. None of these (Spontaneous only)

11. Don't know (Spontaneous only)

12. Refusal (Spontaneous only)

ASK IF Q1 = 9

Please specify your condition (STRING 255)

\section{ASK ALL}

Q2. Are your day-to-day activities limited because of a health problem or disability which has lasted or is expected to last at least 12 months?

1. Yes, limited a lot

2. Yes, limited a little

3. No 